\title{
CHARACTERIZATIONS OF NATURAL SUBMODULAR GRAPHS: A POLYNOMIALLY SOLVABLE CLASS OF THE TSP
}

\author{
YALE T. HERER AND MICHAL PENN
}

(Communicated by Joseph S. B. Mitchell)

\begin{abstract}
Let $G=(V, E)$ be a graph and $w: E \rightarrow R^{+}$be a length function. Given $S \subseteq V$, a Steiner tour is a cycle passing at least once through each vertex of $S$. In this paper we investigate naturally submodular graphs: graphs for which the length function of the Steiner tours is submodular. We provide two characterizations of naturally submodular graphs, an $O(n)$ time algorithm for identifying such graphs, and an $O(n)$ time algorithm for solving the Steiner traveling salesman problem on such graphs.
\end{abstract}

We study the relationship between submodular functions and the length of traveling salesman tours on a graph. Our study was motivated by results in the production/distribution literature. See for example [FQZ92], [Q85], [HR90], [R86], [HP93]. A set function $G(S)$ that maps subsets of $V$ to $\mathfrak{R}$ is said to be submodular if $G(S \cup L \cup M)-G(S \cup L) \leq G(S \cup M)-G(S)$ for all disjoint $S, L, M \subset V$. We use a slightly liberalized definition of a traveling salesman tour called the Steiner traveling salesman tour [CFN85]. For an excellent review of the classical traveling salesman problem see Lawler et al. [LLRS85]. In the Steiner traveling salesman tour on a subset of vertices $S$, the tour must visit the vertices in $S$ at least once, but it may also visit some vertices not in $S$ one or more times. Our Steiner traveling salesman tour differs from the one in [CFN85] since we require it to visit a special vertex called the central warehouse. If for a particular graph the lengths of the Steiner traveling salesman tours are submodular for all nonnegative weight functions and all choices of the central warehouse, then the graph is termed naturally submodular. Note that there are graphs which are not naturally submodular but that the tour lengths are submodular for certain weight functions. We need to include the central warehouse on the Steiner traveling salesman tours because otherwise (assuming the length of the tour through the empty set is defined to be zero) only trivial graphs are naturally submodular.

Received by the editors May 25, 1993; presented during the 15th International Symposium on Mathematical Programming, Ann Arbor, MI, August 15-19, 1994.

1991 Mathematics Subject Classification. Primary 05C38, 05C45, 05C75, 05 C85.

Key words and phrases. Submodular functions, traveling salesman problem, graphs, algorithms.

The first author's research was partially supported by the Center for Absorption in Science, Ministry of Absorption, Israel and by the Technion V.P.R. Fund B.\&L. Bloomfield Industrial Management R. F.

The second author's research was partially supported by Mendes France Fellowship Trust. 
In this paper we present two characterizations of naturally submodular graphs, one based on the number of internally vertex disjoint paths and one on a decomposition. In Herer [H90], graphs that are trees are shown to be naturally submodular. Anily and Federgruen [AF90] give an example of a graph which is not naturally submodular, while an example of a smallest such graph with unit lengths can easily be adapted from [H90]. Herein we demonstrate that naturally submodular graphs can be recognized in $O(n)$ time. In addition, for any $S \subseteq V$, an $O(n)$ algorithm for computing an optimal Steiner tour is presented.

Our results are more applicable when the Steiner tour lengths fit into what is called the family model [HP93]. A set function $\mathscr{F}$ is said to fit into the family model if $\mathscr{F}(S)$ can be written as $\mathscr{F}(S)=\sum\{F(M): M \subseteq V, M \cap S \neq \varnothing\}$ and $F$ is any. set function that maps subsets of $V$ to $\mathfrak{R}^{+}$, where $\mathfrak{R}^{+}$is the set of nonnegative real numbers. Note that every set function that fits into the family model is nonnegative monotone submodular [FZ88].

We assume that the reader is familiar with the basic definitions of graph theory (see for example [BM76]). A path consisting of a single edge is referred to as a trivial path. Let $G=\left(V_{G}, E_{G}\right)$ and $H=\left(V_{H}, E_{H}\right)$ with $V_{G} \cap V_{H}=\varnothing$. Let $v_{g} \in V_{G}$ and $v_{h} \in V_{H}$. We define a new graph called a 1-sum of $G$ and $H$ by identifying $v_{g}$ with $v_{h}$. Let $S \subseteq V$ be a subset of vertices. We denote our special Steiner traveling salesman tour (as described above) by $t(S)$ and its value by $T(S)$. An optimal tour is denoted by $t^{*}(S)$ and its length by $T^{*}(S)$. To insure that the central warehouse is visited on every tour, we include it in the Steiner vertices. Note that $t(S)$ can also be thought of as a concatenation of shortest paths between the vertices on an ordered list containing $S$ and the central warehouse. Observe that these shortest paths might go through vertices in $S$, but that the tour is not said to visit $S$ at these vertices. We assume without loss of generality (because the offending edge can be removed without changing the optimal tour) that the weight function $w$ satisfies the triangle inequality.

\section{ChaRACTERIZATION OF NATURALly SUBMODULAR GRAPHS}

Theorem 1. Let $G=(V, E)$ be a simple connected graph and $w: E \rightarrow \mathfrak{R}^{+} a$ weight function. Then the following conditions are equivalent:

submodularity: $G$ is naturally submodular.

path: There are at most two internally vertex disjoint nontrivial paths between any two vertices of $G$.

decomposition: $G$ may be obtained by means of 1-sums starting from copies of $K_{4}$ and outerplanar graphs.

family: $T(S)$ fits the family model for all nonnegative weight functions $w$ and all choices of the central warehouse.

For convenience each condition mentioned above will be referred to by its label. We prove the theorem by a series of lemmas.

Lemma 1. The submodularity condition implies the path condition.

Proof. We start by showing that the submodularity condition implies the cut condition (as defined below), and then we show that the cut condition implies the path condition. 

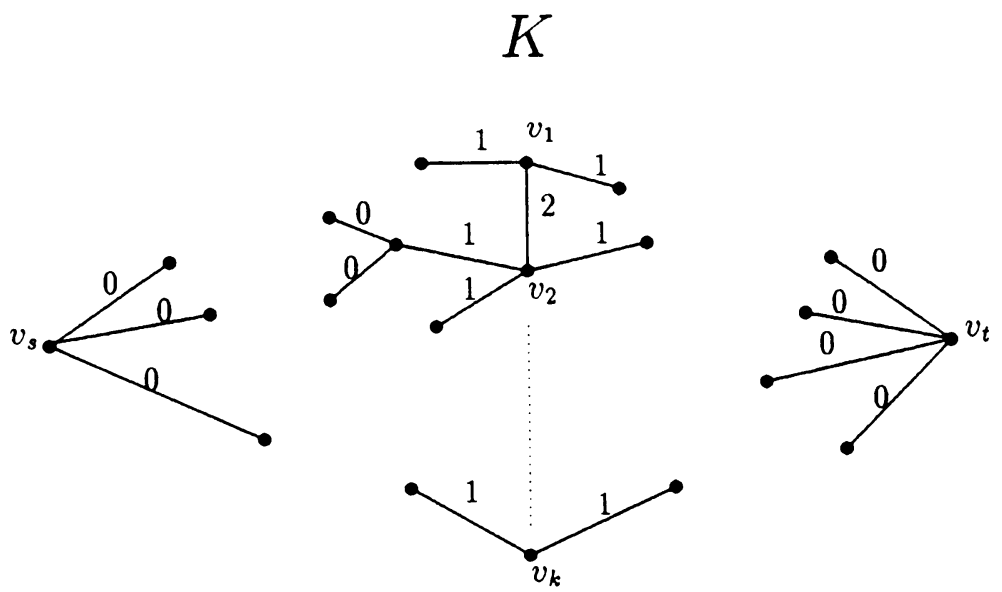

Figure 1. Assignment of weights to the edges.

If $v_{s}$ and $v_{t}$ are two vertices in $V$, then an s-t vertex cut is a set of vertices whose removal together with the edge $\left(v_{s}, v_{t}\right)$, if such exists, from $G$ results in a disconnected graph where $v_{s}$ and $v_{t}$ are not contained in the same component. A minimal s-t vertex cut $K$ is an $s-t$ vertex cut where any proper subset of $K$ is not an $s-t$ vertex cut. The cut condition is: For all pairs of vertices $v_{s}$ and $v_{t}$, every minimal $s-t$ vertex cut set has a cardinality of at most two.

Claim 1.1. The submodularity condition implies the cut condition.

Proof. We prove the claim by demonstrating weights on the edges of any graph with an $s-t$ vertex cut of cardinality greater than two that yield a nonsubmodular $T^{*}(S)$.

For a particular graph that has a minimal $s-t$ vertex cut $K$ of cardinality greater than two, fix $K, v_{s}$, and $v_{t}$. Assign all edges not adjacent to any vertex in $K$ a weight of zero, all edges adjacent to one and only one vertex in $K$ a weight of one, and all edges adjacent to two vertices in $K$ a weight of two. Assign the edge $\left(v_{s}, v_{t}\right)$, if such exists, a weight of two. Pick three vertices in $K$; call them $v_{1}, v_{2}$, and $v_{3}$. Let $v_{t}$ be the central warehouse, $S=\left\{v_{s}, v_{2}\right\}$, $L=\left\{v_{1}\right\}$ and $M=\left\{v_{3}\right\}$ (see Figure 1).

Clearly, $T^{*}(S \cup L \cup M) \geq 6$ and in fact one could show equality. $T^{*}(S)=$ $T^{*}(S \cup L)=T^{*}(S \cup M)=4$ since the minimality of $K$ guarantees that for every vertex in $K$ there is an $s$ - $t$ path that goes through that vertex and not through any other vertex in $K$ and every nontrivial $s$ - $t$ path goes through some vertex in $K$. Thus,

$$
T^{*}(S \cup L \cup M)-T^{*}(S \cup L) \not \leq T^{*}(S \cup M)-T^{*}(S) .
$$

Claim 1.2. The cut condition implies the path condition.

Proof. Assume there are two vertices, $v_{s}$ and $v_{t}$, connected by more than two internally vertex disjoint nontrivial paths. Clearly any $s-t$ vertex cut must meet each one of these paths. Hence, there is a minimal $s-t$ vertex cut with cardinality more than two.

Lemma 2. The path condition implies the decomposition condition. 
Proof. One can easily verify that if $G$ can be obtained by means of 1-sums of $G_{1}, G_{2}, \ldots, G_{k}$, then to prove the theorem it is sufficient to show that the path condition implies that each $G_{i}, i=1, \ldots, k$, is either $K_{4}$ or an outerplanar graph. Clearly, each $G_{i}$ is planar. Assume not; then by Kurotowski's theorem [K30], $G_{i}$ contains either a subdivision of $K_{5}$ or a subdivision of $K_{3,3}$. Thus, there exists two vertices $u$ and $v$ connected by three nontrivial internally vertex disjoint paths. Assume $G_{i}$ is not an outerplanar graph; then every planar embedding will have inner vertices as well as outer vertices. Let $\bar{G}_{i}$ be an embedding of $G_{i}$ for which the number of inner vertices is as small as possible. Let $u$ be an outer vertex which is adjacent to an inner vertex $w$, and determine the subset $W$ of inner vertices which can be reached from $w$ by paths consisting only of inner vertices. If $u$ is the only outer vertex adjacent to vertices in $W$, then the component is not 2-connected. If only two outer vertices $u$ and $v$ are adjacent to vertices in $W$, then $u$ and $v$ are connected by three nontrivial internally vertex disjoint paths: a path from $u$ to $v$ through $W$, and two paths from $u$ to $v$ on the outer face. If $u$ and $v$ were adjacent on the outer face, then we could find an embedding with fewer inner vertices. If there are at least three vertices on the outer face which are adjacent to vertices in $W$, then the component contains either $K_{4}$ or a subdivision of $K_{4}$. If $G_{i}$ is $K_{4}$ we are done, otherwise there are three nontrivial internally vertex disjoint paths connecting two vertices. Thus, if the path condition holds, then $G_{i}$ is either $K_{4}$ or an outerplanar graph.

Definition. Let $t^{*}(V)$ be an optimal tour though $V$. We say that $t^{*}(V)$ or $G$ has the fixed order property if for all $S \subseteq V$ there exists an optimal tour $t^{*}(S)$ that visits the vertices in $S$ in the same order as does $t^{*}(V)$.

One must be careful here, because the fixed order property does not mean the following: If $t^{*}(S)$ is an optimal tour through $S$, then there is a $t^{*}(V)$ that visits the vertices in $S$ in the same order as does $t^{*}(S)$. In fact, this last statement is false, as shown in Figure 2 where all edge lengths are one. Note that the graph in Figure 2 contains a tour $t^{*}(S)$ for which the statement above is true, but for the one presented, $t^{*}(S)=\left(v_{1}, v_{2}, v_{3}, v_{4}\right)$, the statement is false.

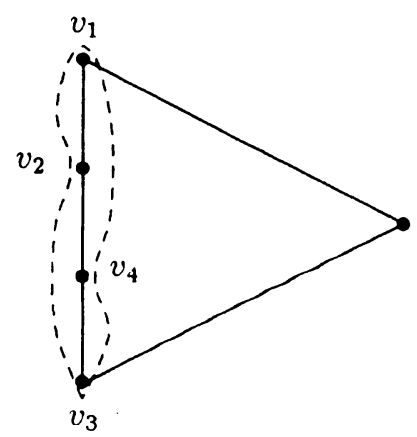

FIGURE 2. A graph with unit edge lengths having an optimal tour $t^{*}(S)$ such that there does not exist an optimal tour $t^{*}(V)$ that visits the vertices in $V$ in the same order as does $t^{*}(S)$. 
Lemma 3. The decomposition condition implies the fixed order property for all nonnegative weight functions and all choices of the central warehouse.

Note that for a given graph the fixed order might be different for different weights.

Proof. Assume the decomposition condition holds; then clearly if the fixed order property holds for each component of $G$, it holds for $G$ as well. One could simply join the tours of the different components at the 1-sum vertices. We must however ensure that the 1-sum vertices are visited by the tours. We do this by including among the Steiner vertices any vertex whose removal would split the graph into two components each having at least one Steiner vertex.

Clearly, $K_{4}$ has the fixed order property, with the order being the optimal tour through $K_{4}$. All that remains to be proven is that any outerplanar graph $G_{i}=\left(V_{i}, E_{i}\right)$ has the fixed order property. Pick a vertex on the outer face and label it 1 . Label the $N$ vertices of $V_{i}$, around the outer face (the direction is irrelevant) $2,3, \ldots, N$. We now show that the tour through a subset $S=$ $\left\{i_{1}, i_{2}, \ldots, i_{z}\right\}$ of $V_{i}$ that visits the vertices in increasing order of their names is optimal. We call such a tour an ordered tour. Note that showing that $G_{i}$ has an optimal tour which is ordered will complete the proof.

The basic idea behind this part of the proof is that any nonordered tour will cross itself, and thus can be uncrossed and made 'closer' to an ordered tour.

Without loss of generality assume that $i_{j}<i_{k}$ if $j<k$. Assume that the ordered tour is not optimal. Let $\hat{t}(S)$ be an optimal tour such that the indices of the vertices in $S$, when arranged in the same order as visited by the tour, is lexicographically minimal. Note that any ordered tour is lexicographically minimal. We name the vertices in $S$ in the same order they are visited in $\hat{t}(S)$ $j_{1}, j_{2}, \ldots, j_{z}$. We know that $i_{1}=j_{1}$ since a tour can begin at any vertex. Due to the fact that this tour is not ordered, it must cross itself at least once; however, the graph is outerplanar, hence these cross(es) must occur at vertices. One can easily uncross $\hat{t}(S)$ and thus obtain a tour of the same length, but lexicographically smaller.

Note that the fixed order through $V$ does not depend on the central warehouse location.

Lemma 4. The fixed order property for all nonnegative weight functions and all choices of the central warehouse implies the family condition.

Proof. Let $v_{0}$ be the central warehouse, and let $v_{0}, v_{1}, v_{2}, \ldots, v_{n}, v_{n+1}=v_{0}$ be an optimal tour through all the vertices and the central warehouse. We define $A_{i j}$ for all $1 \leq i \leq j \leq n$ to be the set $\left\{v_{i}, v_{i+1}, \ldots, v_{j}\right\}$ and $d_{i j}$ to be the length of the shortest path between $v_{i}$ and $v_{j}$. Let $F\left(A_{i j}\right)=d_{i, j+1}+d_{i-1, j}-$ $d_{i, j}-d_{i-1, j+1}$ for all $1 \leq i \leq j \leq n$ and $F(S)=0$ for all $S \neq A_{i j}$ for some $1 \leq i \leq j \leq n$. Theorem 2 of Herer and Roundy [HR90] implicitly shows that if $F\left(A_{i j}\right) \geq 0$ for $1 \leq i \leq j \leq n$, then given the fixed order property these family costs correctly model the value of a tour.

Now we prove that $F\left(A_{i j}\right) \geq 0$. Assume that there exists an $i$ and $j$ such that $F\left(A_{i j}\right)<0$. By the fixed order property, $v_{i-1}, v_{i}, v_{j}, v_{j+1}, v_{i-1}$ is an optimal tour through the set of vertices $\left\{v_{i-1}, v_{i}, v_{j}, v_{j+1}\right\}$ with the central 
warehouse located at $v_{i-1}$. This tour has length

$$
\begin{aligned}
& d_{i-1, i}+d_{i, j}+d_{j, j+1}+d_{j+1, i-1} \\
& >d_{i-1, i}+d_{i, j+1}+d_{j+1, j}+d_{j, i-1} .
\end{aligned}
$$

(2) is less than (1) because we assumed $F\left(A_{i j}\right)<0$ and our path lengths are symmetric. However (2) represents the length of a feasible tour through the set of vertices $\left\{v_{i-1}, v_{i}, v_{j}, v_{j+1}\right\}$ which contradicts the optimality of the tour $v_{i-1}, v_{i}, v_{j}, v_{j+1}, v_{i-1}$. Hence, we known that $F\left(A_{i j}\right) \geq 0$.

Proof of Theorem 1. The proof is straightforward using the previous lemmas and the fact that the family condition implies the submodularity condition (as shown in [FZ88]).

Theorem 2. Let $G=(V, E)$ be a simple connected graph with $|V|=n$. Then, there is an $O(n)$ time algorithm for determining if the graph is naturally submodular.

Proof. The algorithm contains two steps. First one finds the 2-connected components using Tarjan's $O(n)$ algorithm [T72]. Second, for each component which is not $K_{4}$ one determines if the graph is outerplanar by using Mitchell's $O(n)$ algorithm [M79].

Theorem 3. The Steiner traveling salesman problem in naturally submodular graphs can be solved in $O(n)$ time.

Proof. Let $G=(V, E)$ be a graph such that $G$ can be obtained by means of 1-sums of $G_{1}, G_{2}, \ldots, G_{k}$ with $G_{i}=\left(V_{i}, E_{i}\right)$. Let $S \subseteq V$ and $S_{i}=S \cap V_{i}$, $i=1, \ldots, k$. Given optimal tours $t_{i}^{*}\left(S_{i}\right)$ through $G_{i}$ for all $i$, it is obvious how to construct an optimal tour $t^{*}(S)$ through $G$. We only mention here that the edges in $t^{*}(S)$ are simply the union of the edges in all of the $t_{i}^{*}\left(S_{i}\right)$ 's.

Cornuéjols, Fonlupt, and Naddef [CFN85] show that the Steiner traveling salesman problem can be solved in linear time for series-parallel graphs. Since the problem is trivial for $K_{4}$ and outerplanar graphs are series-parallel, the proof is completed by using our decomposition characterization.

\section{REFERENCES}

[AF90] S. Anily and A. Federgruen, One warehouse multiple retailer systems with vehicle routing costs, Management Sci. 36 (1990), 92-114.

[BM76] J. A. Bondy and U. S. R. Murty, Graph theory with application, Elsevier Science, New York, 1976.

[CFN85] G. Cornuéjols, J. Fonlupt, and D. Naddef, The Traveling Salesman Problem on a graph and some related polyhedra, Math. Programming 33 (1985), 1-27.

[FZQ92] A. Federgruen, M. Queyranne, and Y. S. Zheng, Simple power of two policies are close to optimal in a general class of production/distribution networks with general joint setup costs, Math. Oper. Res. 17 (1992), 951-963.

[FZ88] A. Federgruen and Y.S. Zheng, Minimizing submodular set functions: Efficient algorithms for special structures with applications to joint replenishment problems, Working Paper, Wharton School, University of Pennsylvania, Philadelphia, 1988.

[H90] Y. Herer, Submodularity and the Traveling Salesman Problem, Tech. Report No. 915, School of Operations Research and Industrial Engineering, Cornell University, Ithaca, NY, 1990. 
[HP93] Y. Herer and M. Penn, Characterizations of naturally submodular graphs: motivational and algorithmic aspects, Operations Research Statistics and Economics Mimeograph Series No. 407, Industrial Engineering and Management, Technion-Israel Institute of Technology, Haifa, Israel, 1993.

[HR90] Y. Herer and R. Roundy, Heuristics for a one warehouse multi-retailer distribution problem with performance bounds, Oper. Res. (to appear).

[K30] C. Kuratowski, Sur le probleme des courbes gauches en topologie, Fund. Math. 15 (1930), 271-283.

[LLRS85] E. L. Lawler, J. K. Lenstra, A. H. G. Rinnooy Kan, and D. B. Shmoys (eds.), The Traveling Salesman Problem, a guided tour of combinatorial optimization, Wiley, Chichester, 1985.

[M79] S. L. Mitchell, Linear algorithms to recognize outerplanar and maximal outerplanar graphs, Inform. Process. Lett. 9 (1979), 229-232.

[Q85] M. Queyranne, A polynomial-time submodular extension to Roundy's 98\%-effective heuristic for production/inventory systems, Working Paper No. 1135, Faculty of Commerce and Business Administration, University of British Columbia, Vancouver, 1985.

[R86] R. Roundy, A 98\%-effective lot-sizing rule for a multi-product, multi-stage production/ inventory system, Math. Oper. Res. 11 (1986), 699-727.

[T2] R. Tarjan, Depth-first search and linear graph algorithms, SIAM J. Comput. 1 (1972), 146-160.

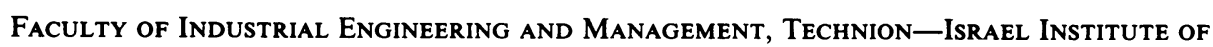
TEChNOLOGY, Haifa, 32000 IsRael

E-mail address: yale@techunix.technion.ac.il

E-mail address: mpenn@ie.technion.ac.il 